\section{Visión Electrónica Más que un estado sólido \\ https://doi.org/10.14483/issn.2248-4728}

UNIVERSIDAD DISTRITAL

FRANCISCO JOSÉ DE CALDAS

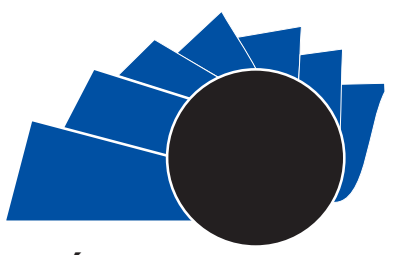

VISIÓN ELECTRONICA

Visión investigadora

\title{
Planeación de trayectorias por Fuzzy C-means para robots móviles
}

\author{
Planning of pathways by Fuzzy C-means for mobile robots
}

Paula Catalina Useche Murillo ${ }^{\prime}$, Javier Orlando Pinzón Arenas ${ }^{2}$, Robinson Jiménez Moreno ${ }^{3}$

INFORMACIÓN DEL ARTICULO

Historia del articulo

Enviado: 14/12/2017

Recibido: $17 / 04 / 2018$

Aceptado: 08/05/2018

\section{Palabras clave: \\ Control difuso, \\ Fuzzy C-Means, \\ Planeación de trayectorias, \\ Robot móvil.}

Keywords:

Fuzzy control,

Fuzzy C-Means,

Trajectory planning,

Mobile Robot.

\section{RESUMEN}

En este artículo se presenta el diseño y ejecución de un algoritmo de planeación de trayectorias - en un ambiente de trabajo simulado - empleando el método de clustering por Fuzzy C-Means, para determinar el grado de pertenencia de cada punto del espacio a los obstáculos presentes en el área de desplazamiento de un robot móvil. Se desarrolló una herramienta en MATLABß que permite ingresar la cantidad de objetos en dicha área, y así encontrar todos los posibles caminos que pasen en medio de ellos. El algoritmo logra identificar caminos para un ambiente con máximo 6 obstáculos, y le permite al usuario ingresar datos de control del algoritmo -como el coeficiente de partición del difuso (m) empleado para definir el ancho de cada camino según los requerimientos de la aplicación.

\section{ABSTRACT:}

In this paper presents the development of a trajectory planning algorithm for a simulated work environment, where the Fuzzy C-Means clustering method was used to determine the degree of membership of each space point obstacles presents to robot displacement and to find all possible paths that pass in the middle of them. The algorithm identifies paths for an environment with maximum 6 obstacles, and allows the user to enter the partition coefficient of the fuzzy $(\mathrm{m})$ to define the width of each path according to the requirements of the application.

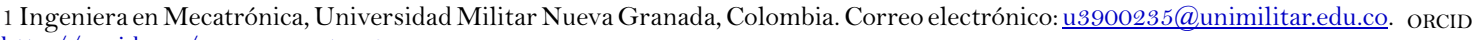
http://orcid.org/0000-0002-7298-6900.

2 Ingeniero en Mecatrónica, Universidad Militar Nueva Granada, Colombia. Esp. En Gerencia Integral de Proyectos de Ingeniería, Universidad Militar Nueva Granada, Colombia. Correo electrónico: u3900231@unimilitar.edu.co. orcid https://orcid.org/0000-0001-8521-2077.

3 Ingeniero Electrónico, Universidad Distrital Francisco José de Caldas, Colombia. MSc. En Automatización Industrial, Universidad Nacional de Colombia. PhD. En Ingeniería, Universidad Distrital Francisco José de Caldas. Docente Universidad Militar Nueva Granada, Colombia. Correo electrónico: robinson.jimenez@unimilitar.edu.co.orcid https://orcid.org/0000-0002-4812-3734.

Citar este artículo como: P. C. Useche-Murillo, J. O. Pinzón-Arenas y R. Jiménez-Moreno, "Planeación de trayectorias por Fuzzy C-Means para robots móviles", Visión electrónica, algo más que un estado sólido, vol. 1, no. 1, Edición especial, enero-junio 2018.DOI revista: https://doi.org/10.14483/issn.22484728 . 


\section{Introducción}

El desplazamiento autónomo de robots móviles dentro de un ambiente no estructurado implica la necesidad de reconocer e identificar todos los posibles obstáculos que debe evadir el móvil durante su recorrido, y poder establecer así un camino accesible para el robot a través del cual pueda desplazarse sin chocar, como se expone en [1]. La finalidad del presente artículo es desarrollar un algoritmo de planeación de trayectorias que le entregue al usuario todos los posibles caminos libres para el desplazamiento del móvil, buscando el distanciamiento máximo entre objetos que le permita la variación de la posición de cada obstáculo y, en cierta medida, la cantidad de estos. Para ello se emplea el algoritmo Fuzzy C-Means mediante el cual se divide el área de trabajo en grupos, según la posición de los obstáculos, y se establecen como trayectorias todos aquellos puntos que pasen por los límites de dichos grupos.

Como antecedentes a la presente investigación la literatura documenta algunas soluciones propuestas para la planeación de trayectorias en robots móviles. La presentada en [1] incluye métodos combinados como el Campo Potencial Artificial y estimaciones de Filtro Kalman Multi-rate con el fin de estimar la ubicación futura de los obstáculos actuales y con ello definir una trayectoria para el móvil. Otra, expuesta en [2], utiliza el movimiento de partículas brownianas donde se combinan campos artificiales y enjambres de partículas para la planeación de trayectorias; y con este mismo fin, en [3] se generan trayectorias curvas y continuas en base a clotóidos - segmentos de línea y arcos circulares -.

Por otro lado, en [4] se generan trayectorias suaves para un móvil no holonómico usando curvas Bézier; en [5] se generan trayectorias suaves, dependientes del tiempo para móviles no holonómicos, empleando polinomios de grado 3 y 5; en [6] se desarrolla un algoritmo de planeación de trayectorias basado en filtrado no lineal, en gestión lógica de entradas de referencia, en restricciones dinámicas y en linealización dinámica; y en [7], se propone controlar el movimiento del robot por medio de la regresión de procesos gaussianos que le permiten al móvil navegar de forma automática en un entorno dinámico.

Con respecto a otros métodos de planeación de trayectorias, en [8] se obtienen múltiples trayectorias en un ambiente no estructurado empleando Fuzzy CMeans y visión de máquina para capturar los elementos del entorno, segmentar los obstáculos, calcular sus centroides y generar las trayectorias. Ese trabajo está muy cerca al aquí expuesto, pero no involucra una herramienta dinámica que permita variaciones del algoritmo, asunto que sí se presenta en el presente trabajo. En [9], en tanto, se hace un análisis de la influencia que genera el coeficiente de partición del Fuzzy (m) encargado de determinar el grado de superposición de los grupos en la generación de cada uno de ellos, llegando a la conclusión de que tanto valores altos como bajos afectan fuertemente el agrupamiento de los datos, aspecto aprovechado en el presente desarrollo para validación de las trayectorias. Finalmente, en [10] se presenta el diseño de un controlador por lógica difusa para robots móviles con restricción de curva limitada.

En la literatura de la Robótica Móvil aparecen múltiples algoritmos difusos evidenciándose la versatilidad de los mismos.

De lo expuesto, el presente artículo se estructura en tres secciones principales: inicialmente la del Algoritmo de identificación de trayectorias, donde se habla sobre la adquisición de datos y el desarrollo del Fuzzy C-Means, con una sección de identificación de trayectorias estableciendo cada una de ellas como subsecciones; luego se presenta la sección de Resultados y Análisis donde se presentan pruebas para una diferente cantidad de obstáculos; finalmente se exponen las conclusiones alcanzadas.

\section{Algoritmo de identificación de trayectorias}

El algoritmo desarrollado le permite al usuario ingresar la cantidad de obstáculos del área de trabajo, la ubicación de cada uno de ellos, y el coeficiente de partición de fuzzibidad para el cálculo del control por el sistema de inferencia difusa. Posteriormente, calcula el grado de pertenencia de cada punto del espacio con respecto a todos los obstáculos y genera caminos entre ellos -donde los puntos cuya función de pertenencia para dos o más obstáculos difiera por menos de un 1,5\%, representan un camino factible para la aplicación-.

Por ejemplo, en la Figura 1 se presenta el reconocimiento de trayectorias para un ambiente simulado donde se incluyen 4 obstáculos ampliamente espaciados entre sí: los rectángulos representan los obstáculos, los puntos sencillos representan el área más próxima a ellos, y los símbolos de tonalidades oscuras los caminos factibles para un coeficiente de partición difusa igual a 25 . 


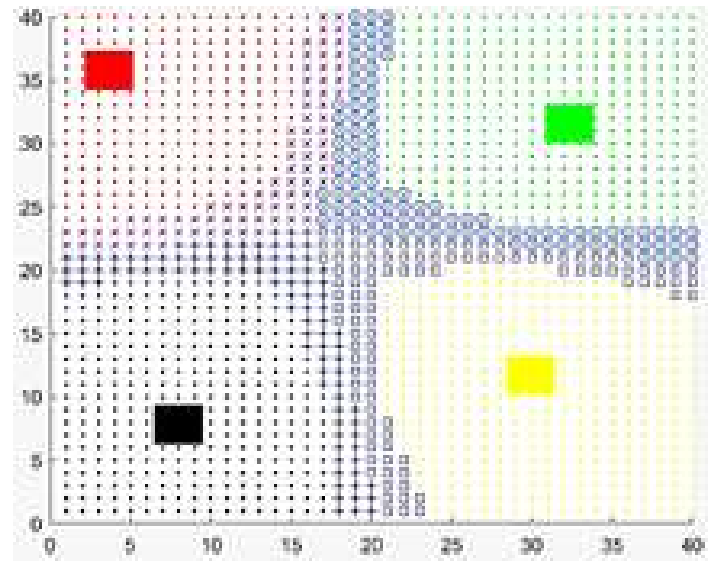

Figura 1. Identificación de trayectorias para un ambiente con cuatro obstáculos.

Fuente: elaboración propia.

\subsection{Adquisición de datos}

La herramienta desarrollada inicia solicitando al usuario ingresar la cantidad de obstáculos y, seguidamente, el coeficiente de partición del Fuzzy; luego se abre una ventana en blanco para el ingreso de los obstáculos, como se muestra en la Figura 2, donde el usuario utiliza el Mouse - representado por cuatro líneas negras en forma de cruz - para seleccionar cualquier parte de la imagen e ingresar el centroide del obstáculo en la posición deseada, el cual se representa con una X

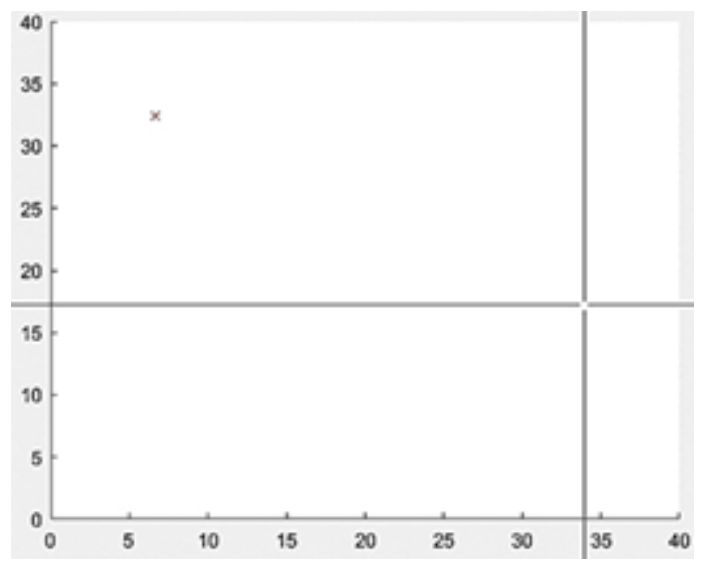

Figura 2. Ingreso manual de obstáculos. Fuente: elaboración propia.

Como se observa en la Figura 2 , el área de trabajo es de 40x40 unidades, y se permiten ingresar los obstáculos en cualquier parte de la superficie.

\subsection{Fuzzy C-Means}

Una vez ingresados los obstáculos, el programa procede a calcular los grados de pertenencia de cada punto con respecto a cada uno de los obstáculos, para lo cual se emplea (1):

$x$ coresponde a los puntos de trabajo,

$c$ a los centroides de los obstáculos.

$m$ al coeficiente de participación difusa

$n \quad$ a la cantidad de obstáculos

$i$ al iterador para los puntos del área de trabajo

kyj a los iteradores para los obstáculos

$u$ ij al valor de pertenencia de cada punto del área de trabajo

$x_{i}$ a cada uno de los obstáculos

$$
u_{i j}=\frac{1}{\sum_{k=1}^{N}\left(\frac{\left\|x_{i}-c_{j}\right\|}{\left\|x_{i}-c_{k}\right\|}\right)^{\frac{2}{m-1}}}
$$

El cálculo del valor de pertenencia de cada punto con respecto a cada uno de los obstáculos $\left(u_{i j}\right)$ mediante (1) se obtiene dividendo la distancia de un punto $x_{i}$ con respecto al centroide del obstáculo deseado $C_{j}$ entre la distancia de ese mismo punto con todos los centroides de los obstáculos $C_{k}$ Luego se debe elevar cada resultado a la $\left(\frac{2}{m-1}\right)$ sumar cada uno de los valores y hacer la división de uno sobre el resultado final, generando un arreglo con todos los valores de pertenencia obtenidos.

Luego, en función de dicho arreglo, se dice que un punto del área de trabajo se encuentra más cerca a aquel obstáculo con respecto al cual su valor de pertenencia es mayor que los demás, y se grafica con el color de dicho obstáculo.

En la Figura 3 se muestran los valores de pertenencia de cada punto del área de trabajo con respecto a todos los obstáculos del ejemplo presentado en la Figura 1. El color de cada señal indica el obstáculo respecto al cual se obtuvo el valor de pertenencia, el eje Y indica el valor de pertenencia de cada punto, y el eje X el punto del área de trabajo evaluado $(40 \mathrm{X} 40=1.600)$ el cual se toma recorriendo todas las filas de una columna antes de pasar a la siguiente. 


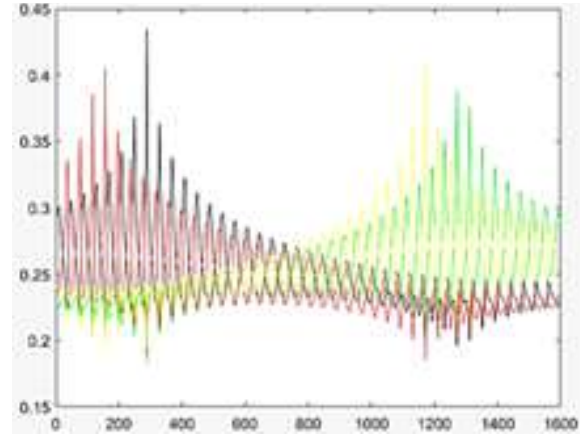

Figura 3. Valores de pertenencia para el área de trabajo de la Figura 1.

Fuente: elaboración propia

Como se puede observar en la Figura 3, a medida que un punto se aleja de un obstáculo cercano su valor de pertenencia respecto a dicho obstáculo se reduce, y a medida que se acerca, como era de esperarse, se incrementa. En la Figura 4a se presenta un ejemplo de planeación de trayectorias para 2 obstáculos, donde es posible observar con mayor claridad los valores de pertenencia de cada punto para ambos obstáculos; en la Figura 4b, los puntos más cercanos al lado izquierdo de la gráfica tienen mayor pertenencia al obstáculo negro, y los de la derecha al otro obstáculo (verde).

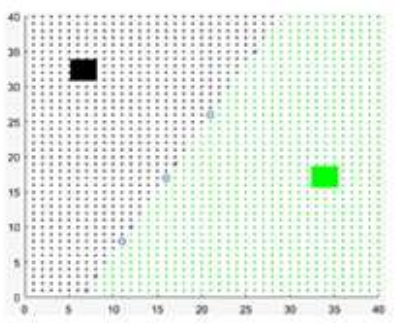

(a)

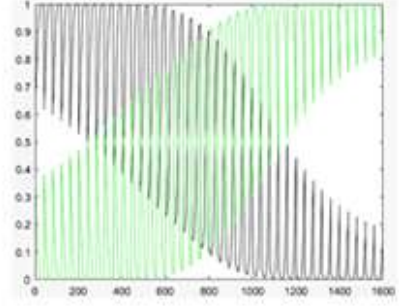

(b)
Figura 4. Planeación de trayectorias para (a) un ambiente con dos obstáculos y (b) los valores de pertenencia del área de trabajo. Fuente: elaboración propia.

\subsection{Identificación de trayectorias}

Una vez que se divide el área de trabajo en grupos, se procede a escoger todas las posibles trayectorias libres de obstáculos, las cuales se seleccionan sobre los límites que dividen cada uno de los grupos. Las características de dichos limites destacan por tener valores de pertenencia, con respecto a todos los obstáculos, muy cercanos entre sí, donde a medida que el coeficiente $\mathrm{m}$ aumenta los valores de pertenencia en los límites, se parecen más y existe una mayor interconexión entre los grupos ya que un mismo punto va a pertenecer en gran medida a más de un obstáculo. En base a la diferencia existente entre los valores de pertenencia de cada punto respecto a cada obstáculo, se seleccionan los caminos para el desplazamiento de un móvil, escogiendo aquellos cuya diferencia es inferior a un valor umbral igual a 0,015 .

En la Figura 5 se comparan dos ejemplos de planeación de trayectorias para un ambiente simulado con tres obstáculos, donde se pueden observar los diferentes anchos de los caminos propuestos para valores de partición de fuzzibidad de 3, para la Figura 5 a, y de 20, para la Figura 5b. Al comparar ambos resultados, se logra identificar la existencia de un camino entre los dos obstáculos de la izquierda para el caso de la Figura 5b con un coeficiente de partición del Fuzzy igual a 20, mientras que para un coeficiente $m$ igual a 3 , dicho camino deja de existir, como muestra la Figura 5a, y los caminos trazados en medio de los obstáculos más alejados tienen un trayecto bastante estrecho entre ellos, a pesar de la distancia.

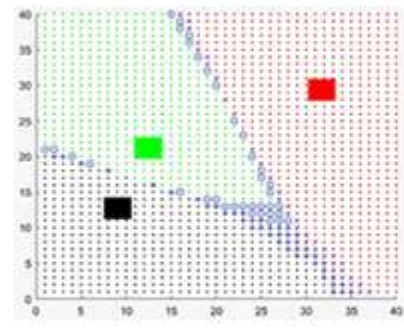

(a)

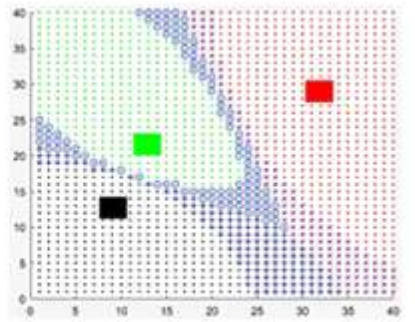

(b)
Figura 5. Planeación de trayectorias para un ambiente con 3 obstáculos. Fuente: elaboración propia.

\section{Resultados y análisis}

En la Figura 6 se presenta un ejemplo de planeación de trayectorias para un ambiente simulado con una cantidad de obstáculos que oscila entre 2 y 6 , para dos valores de partición difusa diferentes, de 5 y 25 . Como se puede observar, los grupos se encuentran fuertemente separados cuando el coeficiente de partición difusa es bajo, lo que genera trayectorias estrechas entre los obstáculos, mientras que para un coeficiente de partición del Fuzzy más alto, las trayectorias son más marcadas y pasan más cerca de los obstáculos, sin tocarlos.

El coeficiente de partición difusa, por ende, está ligado a las dimensiones del móvil y la cantidad mínima de espacio libre que se requiere para que el móvil pueda desplazarse, sin chocar. Si se requiere un amplio rango 
o de movimiento, por ejemplo un móvil voluminoso, el coeficiente debe ser cercano o superior a 20, sino debe ser cercano a 1 .

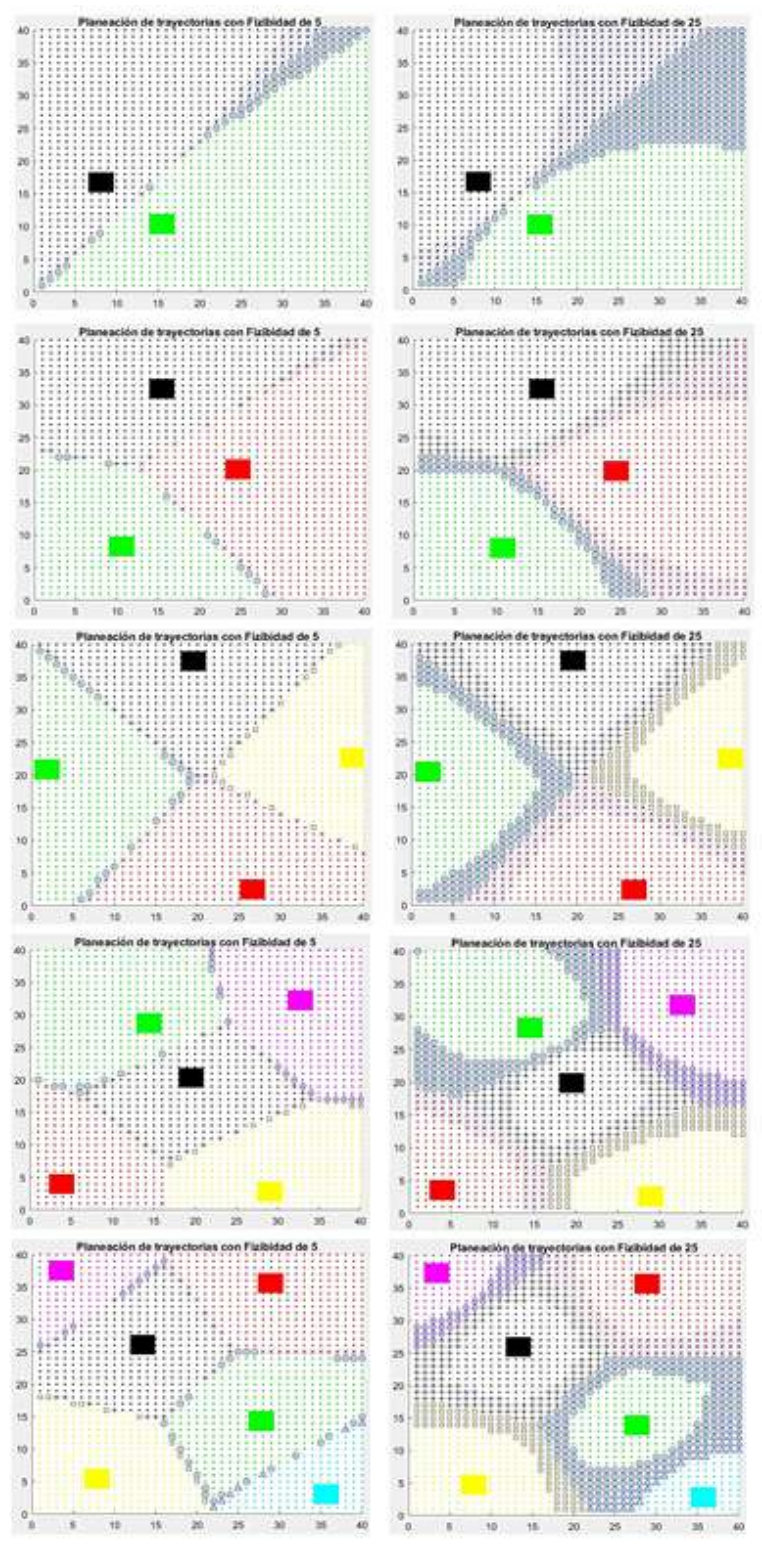

Figura 6. Planeación de trayectorias para diferentes cantidades de obstáculos. Fuente: elaboración propia.

\section{Conclusiones}

El algoritmo de planeación de trayectorias por fuzzy C-Mean permite detectar múltiples trayectorias en un entorno con diferente cantidad de obstáculos, lo que le entrega al robot móvil una amplia gama de caminos por los cuales desplazarse. Adicionalmente, el algoritmo presenta la ventaja de poder definir trayectorias entre obstáculos de cualquier geometría, ya que toda la planeación se hace con respecto al centroide del obstáculo y no a su geometría.

El coeficiente de partición difusa (m) debe seleccionarse de acuerdo a los requerimientos de la aplicación y a las dimensiones del móvil, ya que escoger un valor muy bajo podría generar caminos demasiado estrechos para ser factibles, y un valor demasiado alto, podría presentar caminos lo suficientemente anchos como para que el móvil alcance a tocar los obstáculos. Sin embargo, a pesar de elevar el coeficiente de partición del Fuzzy hasta 25, ninguno de los caminos presentó choques contra ningún obstáculo, sin importar que tan cerca se encontraran entre sí, lo que demuestra la efectividad del algoritmo al momento de buscar caminos que pasen justo en medio de los obstáculos.

Si bien se validó el algoritmo para robots móviles, la proyección de un espacio de trabajo con un robot fijo tipo brazo, donde se desee evitar la colisión del gripper al desplazarse, también puede enmarcarse bajo el algoritmo presentado.

\section{Reconocimientos}

Los autores agradecen a la Universidad Militar Nueva Granada, la cual mediante su vicerrectoría de investigaciones financió el presente proyecto mediante el código IMP-ING-2290 y titulado "Prototipo de robot asistencial para labores de cirugía".

\section{Referencias.}

[1] M. C. Mora y J. Tornero, "Path planning and trajectory generation using multi-rate predictive artificial potential fields", Intelligent Robots and Systems IROS, IEEE/RSJ International Conference on. IEEE, 2008, pp. 2990-2995. DOI: https://doi.org/10.1109/IROS.2008.4651091.

[2] H. E. Espitia y J. I. Sofrony, "Path planning of mobile robots using potential fields and swarms of Brownian particles", Evolutionary Computation (CEC), IEEE Congress, 2011, pp. 123-129. DOI: https://doi.org/10.1109/CEC.2011.5949608. 
[3] V. Girbés, L. Armesto y J. Tornero, "Continuous-curvature control of mobile robots with constrained kinematics", IFAC Proceedings Volumes, vol. 44, no 1, 2011, pp. $\begin{array}{llllllllllllll} & 5 & \mathrm{O} & 3 & - & 3 & 5 & \mathrm{O} & 8 & \text {. } & \mathrm{D} & \mathrm{O} & \mathrm{I} & \text { : }\end{array}$ https://doi.org/10.3182/20110828-6-IT1002.02252 .

[4] K. R. Simba, N. Uchiyama y S. Sano, "Realtime smooth trajectory generation for nonholonomic mobile robots using Bézier curves", Robotics and Computer-Integrated Manufacturing, vol. 41, 2016, pp. 31-42. DOI: https://doi.org/10.1016/j.rcim.2016.02.002.

[5] M. Hashim, M. Sani y T. Lu, "Time-dependent motion planning for nonholonomic mobile robots", IFAC Proceedings Volumes, vol. 42, no 16,2009, p p. $517-522$. D O I : https://doi.org/10.3182/20090909-4-JP2010.00088.

[6] M. Bonfè, C. Secchi y E. Scioni, "Online trajectory generation for mobile robots with kinodynamic constraints and embedded control systems", IFAC Proceedings Volumes, vol. 45, no 22, 2012, pp. 629-635. DOI: https://doi.org/10.3182/20120905-3-HR2030.00062.

[7] S. Choi, E. Kim, K. Lee y S. Oh, "Real-time nonparametric reactive navigation of mobile robots in dynamic environments", Robotics and Autonomous Systems, vol. 91, 2017, pp. 1124 . D O I :

https://doi.org/10.1016/j.robot.2016.12.003.

[8] R. Jiménez y D. J. Lopez, "Trajectory planning for a robotic mobile using fuzzy c-means and machine vision", Symposium of Signals, Images and Artificial Vision - 2013: STSIVA, 2013. DOI :

https://doi.org/10.1109/STSIVA.2013.664 4912.

[9] N. R. Pal y J. C. Bezdek, "On cluster validity for the fuzzy c-means model", IEEE Transactions on Fuzzy systems, vol. 3, no 3, 1995, pp. 370379 . DO I :

https://doi.org/10.1109/91.413225.

[10] G. P. Moustris y S. G. Tzafestas, "Switching fuzzy tracking control for mobile robots under curvature constraints", Control Engineering Practice, vol. 19, no 1, 2011, pp. 45-
5 3. D O I :

https://doi.org/10.1016/j.conengprac.2010. 08.00 . 